\title{
GROUND-BASED AND HST DIRECT IMAGING OF HLQS
}

\section{J. SURDEJ}

STScI, 3700 San Martin Drive, Baltimore, MD 21218, USA, Member of the Astrophysics Division, Space Science Department of the European Space Agency, and Directeur de Recherches au FNRS, Belgium

A.O. JAUNSEN

Nordic Optical Telescope, Ap. 474, S/C de La Palma, E-38700 Canarias, Spain

\section{J.-F. CLAESKENS}

European Southern Observatory (Chile) and Aspirant au FNRS Institut d'Astrophysique, Université de Liège, Avenue de Cointe 5, B-4000 Liège, Belgium

S. GONZAGA AND A. POSPIESZALSKA-SURDEJ

STScI, 3700 San Martin Drive, Baltimore, MD 21218, USA

\section{B. PIRENNE}

ST-ECF, c/o ESO, Karl-Schwarzschild Str. 2, D-85748 Gar-ching bei München, Germany

\section{AND}

\section{A. PRIETO}

MPI für Extraterrestrische Physik, Giessenbachstrasse, D-85748 Garching bei München, Germany

\begin{abstract}
In the context of our studies on gravitational lensing effects among Highly Luminous Quasars (HLQs), we are presently compiling at STScI an archive of direct CCD frames for more than 1000 bright quasars observed with HST and ground-based telescopes. This archive will soon become publicly accessible through the Internet. On the basis of these observations, we are pursuing in a systematic way the analysis (subtraction of numerical PSFs and/or deconvolution) of the HLQ images in order to detect multiple QSO images and/or nearby foreground galaxies at very small angular separations. Residual images corresponding to several new possi-
\end{abstract}



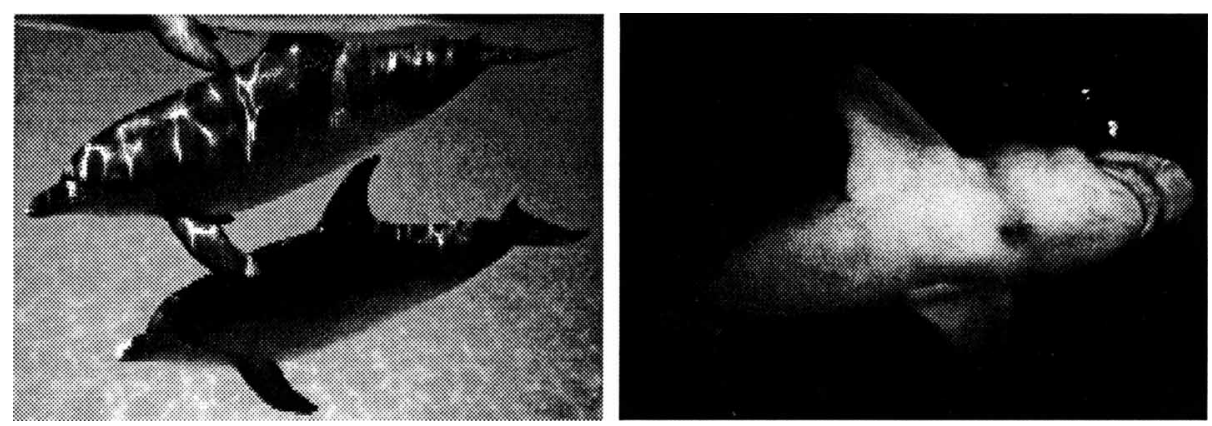

Figure 1. Sunlight caustics projected on dolphins (a) and a shark (b) observing multiple images of a background source (February 1994, Sea World in Orlando, Florida)

ble multiply imaged HLQs are presented here. From the observed number and image configuration of gravitational lens candidates identified in this large sample of HLQs, it is possible to infer realistic values for parameters characterizing the galaxy deflectors, the number counts of quasars, etc. (cf. Claeskens et al. 1995ab), and also to set interesting constraints on the cosmological density of compact objects in the mass range $10^{10}-10^{12} M_{\odot}$.

\section{Preamble: dolphins, sharks, caustics and multiple images}

Figure 1a illustrates very well defined caustics projected on the bodies of swimming dolphins at Sea World (Orlando, Florida). Of course, these caustics result from the complex lensing of sunlight at the wavy interface between air and water. In Fig. 1b, we have reproduced a photograph showing the multiple images of a background source as seen by a shark passing very near such a caustic. There is thus little doubt that dolphins, sharks and many other fishes had witnessed the formation of multiple images of distant background sources -namely the Sun and the Moon- well before 1979. Because of the similarity between the size of these caustics and the distance between the eyes of the big fishes, these animals are also probably aware of the parallax effect. However, it is highly unlikely that dolphins and sharks have the slightest idea of what a 'time delay' is, so that this very concept solely remains a pure astronomical invention (Refsdal 1964).

\section{Introduction}

Following several interesting discoveries of multiply imaged quasars (cf. UM673 AB, Surdej et al. 1987; H1413+117 A-D, Magain et al. 1988; 1208+1011 AB, Magain et al. 1992; 1009-025 AB, Surdej et al. 1993a) within a sample 
of HLQs (typically $M_{V}<-27$ for $H_{0}=50 \mathrm{~km} \mathrm{~s}^{-1} \mathrm{Mpc}^{-1}$ and $q_{0}=0.5$ ), our group has decided to systemize its search for gravitational lensing effects by carefully reanalyzing all direct CCD frames obtained with the ESO $2.2 \mathrm{~m}$, NTT, NOT, CFH, Pic-du-Midi $2 \mathrm{~m}$ and HST telescopes. We presently have in our hands high angular resolution direct CCD frames for approximately 800 distinct quasars and we should obtain CCD frames for about 200 more HLQs during the coming year (planned observations at the Pic-du-Midi, NOT and also with HST during cycle 5). Let us note that most of the well known gravitational lens (GL) systems have been found from a direct visual examination of ground-based CCD frames. A first exception is the case of $1208+1011 \mathrm{~A}$ and B (angular separation of 0.45 "), identified from ground-based direct images (seeing of 1.2", pixel size 0.41") following an efficient numerical PSF subtraction (Magain et al. 1992). Note that this GL has been independently detected in the HST quasar snapshot survey (Bahcall et al. 1992). Of course, by taking advantage of better angular resolution observations, the identification of sub-arcsec separation GLs among HLQs ought to significantly improve the statistical inference of parameters characterizing galaxy deflectors (cf. the velocity dispersion $\sigma^{*}$ ), counts of quasars and also the geometrical structure of the Universe (see Claeskens et al. 1995ab). Furthermore, because of the well known amplification bias affecting flux limited samples of quasars, one should detect a significant excess of bright $(\mathrm{R}<22)$ foreground galaxies in the vicinity (typically < 3") of HLQs. Such an excess of galaxies has been reported by Van Drom et al. (1993), but its statistical significance needs to be improved further. Finally, the incidence of secondary lensed QSO images detected at very small angular separation from the primary ones may lead to interesting constraints on the cosmological density of compact objects (cf. Surdej et al. 1993b). All these arguments very clearly demonstrate the importance of reanalysing in a systematic way (subtraction of numerical PSF, image decomposition, deconvolution, etc. as opposed to a mere visual examination) the direct CCD frames of all HLQs imaged with ground-based telescopes and HST.

\section{Search for very compact GLs among HLQs}

Claeskens, Jaunsen and Surdej (in these proceedings) describe in detail how gravitational lensing statistics were applied to the direct imagery of a sample of more than $1000 \mathrm{HLQs}$ to constrain the values of galactic parameters, the number counts of quasars and the cosmological constant. In order to efficiently perform such applications, it is not only important to have access to a large sample of quasars but also to make use of observations characterized by an optimal angular selection function (ASF; see 
Surdej et al. (1993b) and Jaunsen et al. (1995) for a more detailed account of the ASFs). In some way, the quality of an ASF does actually measure our ability to resolve two QSO images on a CCD frame having a magnitude difference (resp. an angular separation) that is as large (resp. as small) as possible. An easy way to improve the ASF of a given observation consists of course in subtracting from each HLQ image a numerical point spread function (PSF), constructed from nearby stars selected on the CCD frame. Provided that the PSF is spatially well sampled and that it is characterized by a good signal-to-noise ratio, this method is quite insensitive to the image quality (cf. seeing and/or elongation due to telescope motions, etc.). It is also possible to achieve very accurate relative photometry. If no such comparison stars are available, the ASF will then not only be limited by the seeing and the sampling of the CCD images but also by our visual ability to separate nearby QSO components.

For approximately 200 quasars observed with the NOT, 250 quasars observed at ESO and 50 quasars observed within the HST snapshot survey, it has been possible to define very good numerical PSFs (this work is still in progress). We have proceeded as follows: in addition to a careful reduction of all the ground-based data (through the determination of the bias, RON and gain of the CCDs, test of CCD linearity, bias and flat field corrections), we have determined on each CCD frame appropriate regions for sky estimates (void of objects and cosmic rays). Sky subtraction (modeled by a 2-D polynomial of 2nd degree in $\mathrm{X}$ and $\mathrm{Y}$ ) has then been applied and stars were selected to construct a good numerical PSF. Automatic batches perform accurate numerical PSF subtractions, including a weighting scheme that takes into account the CCD RON, Poisson noise in the sky, stars and the QSO, altogether with bi-quadratic rebinning and recentering of the individual PSF reference stars and HLQ images. This procedure is iterated until we get an optimal PSF (i.e. excluding composite or extended stars, etc.). Special decompositions of HLQ images into multiple components are performed for the interesting cases. In the course of this project, we have realized that all the resulting bias and flat field corrected CCD frames could form a new public archive, since most of these ground-based CCD frames are of good quality (good seeing, good sampling of the PSF, etc.) and could be useful to the whole astronomical community for various other scientific purposes (cf. relative photometry, astrometry, counts of galaxies, etc.). We are thus presently checking, updating or creating FITS parameters, headers, etc. for the CCD frames that will become part of the HLQs archive. This archive should become accessible through the Internet by the end of 1996. We welcome very much observers to contact us in order to possibly integrate their direct CCD frames of bright quasars in the HLQs archive.

From the HST snapshot observations, we have been able to construct 


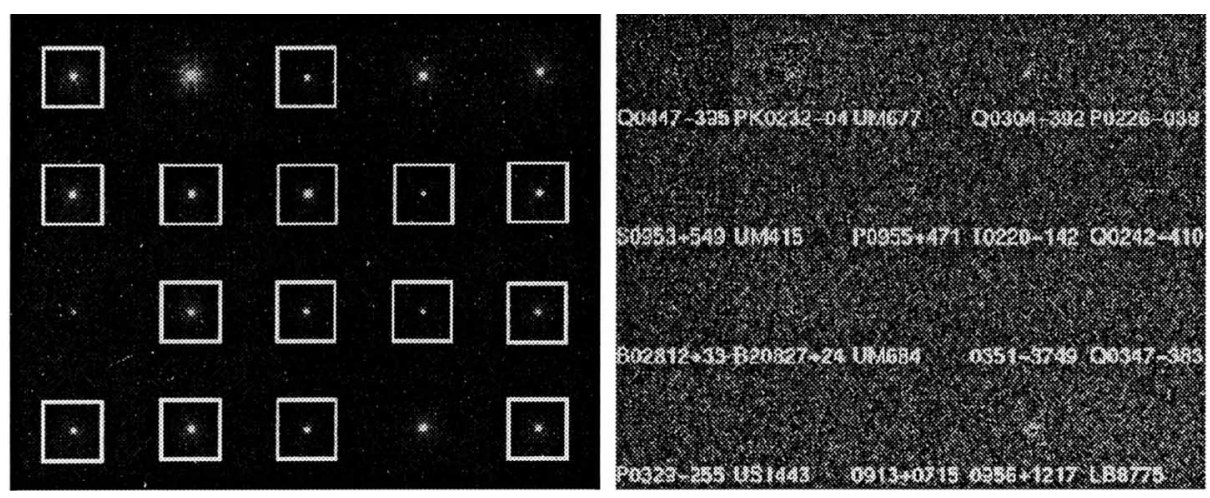

Figure 2. Direct images (a, left) and residuals (b, right) scaled in $\sigma$ unit, for 20 selected HLQs observed with HST. These residuals were obtained by subtracting from each QSO image a scaled numerical PSF constructed from those quasar images surrounded by a large white square (see text)

accurate numerical PSFs for three sets of 23, 20 and 14 quasars imaged at the same location on the CCD planetary camera and observed during time intervals free from any telescope focus readjustment. Fig. 2 illustrates for the set of 20 quasars observed with HST the direct images (a: left) and the residuals (b: right; normalized in $\sigma$ unit) left over after subtracting an average numerical PSF constructed from those quasars surrounded by a large white square. The 19th object in Fig. $2 \mathrm{~b}$, identified as $0956+1217$, displays very significant residuals possibly due to the superposition of a foreground galaxy. Similar analyses of ESO (Fig. 3a) and NOT (Fig. 3b) observations have revealed several interesting gravitational lens candidates with very small angular separations. The residuals shown in Figs. 3ab are very symptomatic of double point-like images. These GL candidates will be further studied with HST during cycle 5 .

\section{New constraints on the density of compact objects}

In order to significantly improve existing upper limits on the density $\Omega_{L}$ (measured in units of the critical density $\Omega_{0}$ ) of putative lensing compact objects in the Universe with mass $M_{L}$, we have followed Surdej et al. (1993b) and made use of the observed number of multiply imaged quasars ( 5 confirmed GLs and 6 other candidates; cf. Figs. 3ab for two of the latter ones) in the sample of 1178 HLQs assembled by Claeskens et al. (1995ab) as well as of the observed angular separation $(\leq 3$ ") and magnitude difference $(\leq 5)$ between their multiple images. At a $99.7 \%$ confidence level for $\mathrm{H}_{0}=$ $50 \mathrm{~km} \mathrm{sec}^{-1} \mathrm{Mpc}^{-1}, \Omega_{0}=1$ and $\Lambda=0$, we find that $\Omega_{L} \leq 0.017,0.005$ and 0.018 for $M_{L}=10^{10}, 10^{11}$ and $10^{12} M_{\odot}$, respectively. These are presently 


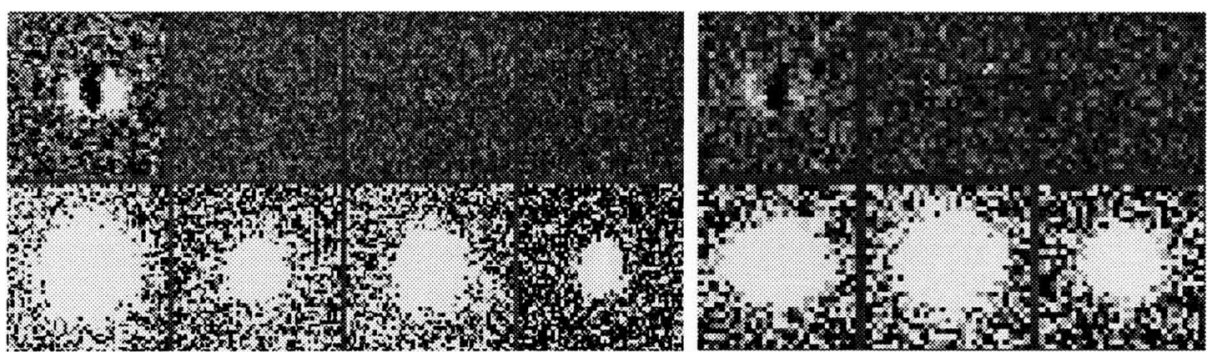

Figure 3. Direct images (lower row) and residuals (upper row) scaled in units of $\sigma$, for the new GL candidate J03.13 (a, left) identified by Claeskens et al. (1995c and d) at ESO and another GL candidate (b, right) observed with the NOT. In the latter case, the HLQ has been found to be compatible with two point-like components separated by 0.56 " and a magnitude difference of 0.2 . The direct images (lower row) and residuals (upper row) of the PSF reference stars are shown on the right side of their respective HLQ ones.

the best constraints existing on $\Omega_{L}$ in the mass range $10^{10}-10^{12} M_{\odot}$.

\section{References}

Bahcall, J.N., Hartig, G.F., Jannuzi, B.T., Maoz, D., \& Schneider, D.P., 1992, ApJL, 400, L51

Claeskens, J.-F., Jaunsen, A., \& Surdej, J., 1995a, in preparation

Claeskens, J.-F., Jaunsen, A., \& Surdej, J., 1995b, in these proceedings

Claeskens, J.-F., Surdej, J., \& Remy, M., 1995c, A\&A, submitted

Claeskens, J.-F., Surdej, J., \& Remy, M., 1995d, in these proceedings

Jaunsen, A.O., Jablonski, M., Pettersen, B.R., \& Stabell, R., 1995, A\&A, in press

Magain, P., Surdej, J., Swings, J.P., Borgeest, U., Kayser, R., Kühr, H., Refsdal, \& S., Remy, M., 1988, Nature, 334, 325

Magain, P., Surdej, J., Vanderriest, C., Pirenne, B., \& Hutsemékers, D., 1992, A\&A, 253, L13, (Erratum in A\&A, 272, 383)

Refsdal, S., 1964, MNRAS, 128, 307

Surdej, J., Magain, P., Swings, J.P., Borgeest, U., Courvoisier, T.J.-L., Kayser, R., Kellermann, K.I., Kühr, H., \& Refsdal, S., 1987, Nature, 329, 695

Surdej, J., Remy, M., Smette, A., Claeskens, J.-F., Magain, P., Refsdal, S., Swings, J.P., \& Véron, M., 1993a, in Gravitational Lenses in the Universe, eds. J. Surdej, D. Fraipont-Caro, E. Gosset, S. Refsdal and M.Remy, (Liège: Université de Liège) 153

Surdej, J., Claeskens, J.F., Crampton, D., Filippenko, A.V., Hutsemékers, D., Magain, P., Pirenne, B., Vanderriest, C., \& Yee, H.K.C., 1993b, AJ, 105, 2064

Van Drom, E., Surdej, J., Magain, P., Hutsemékers, D., Gosset, E., Claeskens, J.-F., Shaver, P., \& Melnick, J., 1993, in Gravitational Lenses in the Universe, eds. J. Surdej, D. Fraipont-Caro, E. Gosset, S. Refsdal and M.Remy, (Liège: Université de Liège) 301 